\title{
Ausência de efeito do análogo (hCG) do hormônio luteinizante na angiogênese luteal em ratas (Rattus novergicus)
}

\section{Faillure in the effect of the analogue (hCG) of luteinizing hormone on the luteal angiogenesis in rats (Rattus novergicus)}

\section{Diego Gonçalves SILVA ${ }^{1}$; Diego Marcondes GUERRA ${ }^{1}$; Vinícius José Moreira NOGUEIRA ${ }^{1}$; Mariana Andrade TORRES ${ }^{1}$; Diego Feitosa LEAL ${ }^{1}$; Carlos Henrique Cabral VIANA ${ }^{1}$; Caroline MARTINS ${ }^{1}$}

\author{
${ }^{1}$ Medicina Veterinária da PUC Minas, Poços de Caldas - MG, Brasil
}

\begin{abstract}
Resumo
A compreensão dos mecanismos de controle da atividade ovariana é necessária para o sucesso das biotecnologias da reprodução. Embora existam inúmeros trabalhos a respeito da aplicação do hormônio luteinizante (LH) na função ovariana, pouco se sabe sobre a sua influência na morfologia e formação da vasculatura do corpo lúteo (CL). Diante disto, o presente projeto teve como objetivo a quantificação da densidade vascular dos CLs de animais tratados com Gonadotrofina Corionica Humana (hCG) após a ovulação. Para tanto, foram utilizados ratas wistar, cujos CLs foram divididos em dois grupos: (A) tratado com hCG na manhã seguinte a cópula e (B) controle (solução fisiológica a 0,9 $\%$ de $\mathrm{NaCl}$ ). Foram confeccionadas lâminas dos ovários dos animais para a quantificação da densidade vascular. Os resultados obtidos não revelaram diferenças significantes entre a densidade vascular dos grupos tratado e controle.
\end{abstract}

Palavras-chave: LH. hCG. Angiogênese. Corpo lúteo. Ovulação.

\begin{abstract}
The knowledge of the mechanisms that affect the control of the ovarian activity is essential for the success of reproduction biotechnologies. Although a number of studies have been carried out in which the luteinizing hormone (LH) was used to control the ovarian activity, little is known about its influence in the morphology and vascular formation of the corpus luteum, aiming to increase the local blood flow. Thus, the objective of the present experiment was the quantification of the vascular density of corpora lutea (Cls) in animals treated with human chorionic gonadotropin (hCG) just after ovulation. Therefore, eighteen wistar rats were used in this experiment. Eight rats in the treated group and ten rats in the control group. Corpora lutea were divided into two groups: group (A) treated with hCG in the following morning after copulation, and group (B) control animals which received an injection of $0.9 \%$ sodium chloride solution. Ovaries from each group were used for preparation of histological sections for vascular density qualification. No statistical significance was found between the two groups tested.
\end{abstract}

Keywords: LH. hCG. Angiogenesis. Corpus luteum. Ovulation.

\section{Introdução}

O corpo lúteo $(\mathrm{CL})$ é uma glândula transitória que se forma a partir da ovulação e tem como função principal produzir progesterona (P4) para manutenção de uma possível gestação ou regulação do ciclo estral e essa produção hormonal é dependente de um aporte sanguíneo adequado ${ }^{1}$.

Os mecanismos controladores da função luteal ainda não foram totalmente elucidados. Em inúmeras espécies, o hormônio luteinizante (LH) é o principal indutor de ovulação e desempenha um papel chave na estimulação e manutenção da produção de $\mathrm{P} 4$ pelo $\mathrm{CL}^{2}$, principalmente na fase final do estro e começo de diestro do ciclo estral ${ }^{3}$. Além do mais, fortes

Correspondência para:

Carlos Henrique Cabral Viana

R. Padre Francis Cletus Cox, 1661 - Jd. Country Club

Poços de Caldas - MG

CEP: 37701-355

e-mail: cabral@biodux.com.br

Recebido: 09/11/2012

Aprovado: 10/04/2013 
evidências indicam a participação do LH no controle da angiogênese luteal ${ }^{2}$.

O processo angiogênico é definido como a formação de novos vasos sanguíneos pela migração e proliferação de células endoteliais oriundas de vasos pré-existentes, sendo um evento essencial para o desenvolvimento luteínico. Durante esse processo, há um equilíbrio entre os fatores que promovem e inibem o desenvolvimento do $\mathrm{CL}^{4}$. Sabe-se que existe uma íntima relação entre vascularização desta estrutura seguida do aumento do fluxo sanguíneo e a produção hormonal $^{5}$. Em ratas, o maior desenvolvimento vascular luteal ocorre 10 a 16 horas após a ovulação ${ }^{1}$.

O corpo lúteo apresenta uma rede capilar sinusóide, em que cada capilar é cercado por três a seis células luteínicas, deste modo, quase todas as células luteínicas estão em contato com um ou mais capilares $^{5,6}$. Quando os capilares são observados ao microscópio eletrônico de varredura, eles apresentam projeções laterais correspondentes ao vazamento da resina pelas fenestrações dos vasos o que comprova a alta permeabilidade vascular (75\% dos capilares do CL são fenestrados) ${ }^{7}$.

Após a angiogênese, o CL se torna um órgão muito vascularizado, recebendo um aporte sanguíneo enorme $^{8}$. Por ocasião da luteólise, formam-se anastomoses arteriovenosas no corpo lúteo de ratas, reduzindo o fluxo sanguíneo ${ }^{7}$. A função lútea inadequada foi associada à diminuição da vascularização lútea, e vários pesquisadores têm sugerido que a diminuição do fluxo sanguíneo do ovário juntamente com a queda de $\mathrm{P} 4$ na circulação sistêmica desempenha um crítico papel na regressão luteal ${ }^{9,10,11}$. Foi constatado que a queda na $\mathrm{P} 4 \mathrm{com}$ o aumento transitório no fluxo sanguíneo levam à luteólise ${ }^{12}$. O corpo albicans possui poucos vasos sanguíneos, e foi considerado por alguns autores como avascular ${ }^{7}$.

A produção inadequada de $\mathrm{P} 4$ é uma das principais causas de infertilidade e morte embrionária ${ }^{4}$. Para uma ampla compreensão dos eventos endócrinos do CL, são necessárias algumas informações sobre os fatores envolvidos e as suas respectivas funções. A preservação da função luteal após a fecundação por mecanismos anti-luteolíticos fisiológicos é necessária para a manutenção da gestação em sua fase inicial. Dessa forma, acredita-se que o conhecimento aprofundado da fisiologia luteal, além de apresentar um valor intrínseco como conhecimento básico, também pode contribuir para a reprodução animal em seu aspecto aplicado.

Sendo assim, o conhecimento da angiogênese luteal em ratas abre a possibilidade para o desenvolvimento de novas metodologias aplicadas ao tratamento de doenças dependentes da angiogênese e inclusive para a identificação de agentes terapêuticos. Embora existam muitos trabalhos referentes à aplicação do LH como indutor da ovulação, até o momento não se encontra na literatura um estudo especializado a respeito do envolvimento da função do LH na estimulação da angiogênese luteal em ratas. Diante deste fato, o presente trabalho teve como objetivo a quantificação da densidade vascular de ratas tratadas com gonadotrofina coriônica humana (hCG) após a ovulação. A hipótese testada seria de que o uso de LH após a ovulação aumentaria a densidade vascular.

\section{Materiais e Métodos}

Os experimentos conduzidos nas dependências no hospital veterinário do campus da PUC Minas de Poços de Caldas foram executados em ratas adultas (Rattus novergicus), da linhagem wistar, em grupos de 10 (dez) animais por tratamento, mantidos em gaiolas apropriadas, com alimentação ad libitum e sob exposição de $12 \mathrm{~h}$ com luz e $12 \mathrm{~h}$ sem luz sob uma temperatura de $22^{\circ} \mathrm{C}$ controlada por condicionador de ar.

Todos os animais foram acompanhados por citologia vaginal por 14 dias para constatação da ciclicidade, em seguida, foram separados em grupos 
por fase do ciclo estral. Os animais com ciclicidade regular foram utilizados no experimento, os demais foram utilizados para a reprodução e manutenção do biotério. Na manhã em que as fêmeas se encontravam no proestro, elas foram separadas, e à noite foram colocadas nas gaiolas dos machos para serem cobertas e induzidas naturalmente a ovulação. As fêmeas cobertas apresentaram espermatozoides na citologia vaginal (D1).

Os animais foram divididos em dois grupos, sendo um grupo tratado com hCG (Grupo A) e um controle (Grupo B):

(A) 10 U.I de hCG (vetecor ${ }^{\oplus}$, Hertape,Brasil) via intraperitoneal

(B) controle (solução fisiologia a $0,9 \%$ de $\mathrm{NaCl}$ ) via intraperitoneal

As aplicações foram realizadas impreterivelmente às 8 horas da manhã do D1.

Na manhã do dia 4 (D4), os animais foram novamente avaliados por citologia vaginal para a verificação de um possível retorno da ciclicidade por ação da progesterona, em seguida foram sedados por inalação de halotano, seguido da aplicação de Tiopental na dose de $40 \mathrm{mg} /$ $\mathrm{kg}$ por via intraperitoneal. Foram eutanasiadas pelo método da guilhotina e ovariectomizadas. Os ovários foram fixados em formol 10\% tamponado, desidratados com álcool, diafanizados com xilol, incluídos em parafina, cortados $(5 \mu \mathrm{m})$ em micrótomo Leica ${ }^{\oplus}$ para confecção das lâminas coradas por Hematoxilina e Eosina (Timm, 2005), com o objetivo de quantificar a densidade vascular. As lâminas foram fotografadas em microscopia de luz, utilizando-se a objetiva de 100X, sobrepostas à fotografia de uma lâmina milimetrada (efetuada com o mesmo microscópio e mesma objetiva). Foram realizadas dez repetições por tratamento de diversos campos em lâminas distintas do mesmo ovário. A contagem dos vasos foi realizada no computador, com sobreposição da imagem da lâmina milimetrada à imagem obtida no corte histológico, determinando-se a densidade vascular por $\mathrm{mm}^{2}$.

\section{Resultados e Discussão}

Os resultados da contagem do número de vasos sanguíneos obtidos foram avaliados estatisticamente pelo teste F, comparando-se as médias dos grupos tratados e controle (Tabela 1).

Apesar dos corpos lúteos das ratas tratadas com hCG serem numericamente mais vascularizados que os das ratas do grupo controle, não houve diferença significante $(\mathrm{p}>0,05)$ entre os grupos. Se o número de animais por grupo fosse maior, possivelmente os resultados seriam diferentes.

A comparação entre corpos lúteos de diferentes ovulações pode ter influenciado o resultado, assim como a densidade vascular folicular antes da ovulação, o tamanho da cavidade folicular e a intensidade da hemorragia, o que sugere a existência de uma diferença fisiológica individual da angiogênese luteal entre os corpos lúteos de indivíduos da mesma espécie.

Tabela 1 - Número médio de vasos sanguíneos por $\mathrm{mm}^{2}$, avaliados em lâminas com cortes histológicos de corpos lúteos de ratas dos grupos tratamento (10 UI de hCG) e controle (Teste F, p=0,31) - Poços de Caldas - 2012

\begin{tabular}{cc}
\hline Tratamento & Número de vasos sanguíneos por $\mathbf{~ m m}^{\mathbf{2}}$ \\
\hline Controle $(\mathrm{n}=10)$ & $19,48 \pm 5,32$ \\
10 UI de hCG $(\mathrm{n}=8)$ & $22,92 \pm 7,65$ \\
\hline
\end{tabular}




\section{Conclusão}

A administração do análogo $(h C G)$ do hormônio lutenizante, na dosagem utilizada, não influenciou angiogênese luteal em ratas (Rattus novergicus).

\section{Referências}

1. MOURA, C. E. B. Expressão do VEGF e vascularização do corpo lúteo em búfalos. 2003. 122 f. Dissertação (Mestrado) Faculdade de Medicina Veterinária e Zootecnia, Universidade de São Paulo, São Paulo, 2003.

2. HAFEZ, E. S. E.; HAFEZ, B. Reprodução animal. 7. ed. Barueri: Manole, 2004. p. 261-278.

3. BOEHM, N.; PLAS-ROSER, S.; ARON, C. Does corpus luteum function autonomously during estrous cycle in the rat? A possible involvement of $\mathrm{LH}$ and prolactin. Journal of Steroid Biochemistry, v. 90, n. 2, p. 663-670, 1984.

4. BERISHA, B.; SHAMS, D. Ovarian function in ruminants. Domestic Animals Reproduction, v. 29, n. 2, p. 305-317, 2005.

5. REDMER, D. A.; REYNOLDS, L. P. Angiogenesis in the ovary. Society for Reproduction and Fertility, v. 1, n. 3, p. 182-192, 1996.

6. MIYAMOTO, A.; SHIRASUNA, K.; SASAHARA, K. Local regulation of corpus luteum development and regression in the cow: impact of angiogenic and vasoactive factors. Domestic Animal Endocrinology, v. 37, n. 3, p. 159-169, 2009.

7. MURAKAMI, T.; IKEBUCHI, Y.; OHTSUKA, A.; KIKUTA, A.; TAGUCHI, T.; OHTANI, O. The blood vascular wreath of rat ovarian follicle, with special reference to its changes in ovulation and luteinization: a scanning electron microscopic study of corrosion casts. Archive of Histology and cytology, v. 51, n. 4, p. 299-313, 1988.

8. WILTBANK, M. C.; DYSKO, R. C.; GALLAGHER, K. P.; KEYES, P. L. ;Relationship between blood flow and steroidogenesis in the rabbit corpus luteum. Journal Reproduction Fertility, v. 84, n. 2, p. 513-520, 1988.

9. REYNOLDS, L. P.; KILliLEA, S. D.; REDMER, D. A. Angiogenesis in the female reproductive system. FASEB Journal, v. 6, n. 3, p. 886-892, 1992.

10.REYNOLDS, L. P.; KILLILEA, S. D.; GRAZUL-BILSKA, A. T.; REDMER, D. A. Mitogenic factors of corpora lutea. Progress in Growth Factor Research, v. 5, n. 2, p. 159-175, 1994.

11.WISE, T. H.; CATON, D.; THATCHER, W. W.; BARRON, D. H.; FIELDS, M. J. Ovarian function during the estrous cycle of the cow: ovarian blood flow and progesterone release rate. Journal Animal Science, v. 55, n. 3, p. 627-637, 1982.

12. ACOSTA, T. J.; YOSHIZAWA, N.; OHTANI, M.; MIYAMOTO A. Local changes in blood flow within the early and midcycle corpus luteum after prostaglandin $\mathrm{F}(2$ alpha) injection in the cow. Biology Reproduction, v. 66, n. 3, p. 651-658, 2002. 